\title{
Calidad de los sitios web en el sector agroalimentario ecológico y sus factores explicativos: el papel del cooperativismo
}

\author{
Enrique Bernal Jurado, Adoración Mozas Moral, \\ Domingo Fernández Uclés, Miguel Jesús Medina \\ Viruel y Raquel Puentes Poyatos
}

RESUMEN: Los objetivos de este trabajo son examinar en qué medida las empresas españolas del sector de la agricultura ecológica están aprovechando el sitio web como canal de venta, identificar los factores que explican la calidad de estas plataformas virtuales y estudiar la relación de dicha calidad sobre los rendimientos empresariales. Para ello, se ha analizado la website de las empresas de aceite de oliva ecológico españolas, la mayoría de ellas cooperativas, identificando los factores relacionados con la calidad de sus sitios web. Entre dichos factores, se ha prestado una especial atención a la influencia de la forma jurídica cooperativa. Para alcanzar estos objetivos se ha utilizado la técnica QCA (Quality Comparative Analysis) en su variante fuzzy set (fsQCA), junto con otras técnicas estadísticas. Los resultados sugieren que las empresas oleícolas ecológicas, especialmente las cooperativas, presentan una escasa calidad de sus sitios web y que esta calidad está relacionada directamente con la obtención de unos mejores ingresos de explotación. Cabe destacar que, entre los distintos elementos utilizados para valorar la calidad del sitio web, es la información sobre responsabilidad social corporativa la que se relaciona con más intensidad con los resultados empresariales.

PALABRAS CLAVE: Agricultura ecológica, aceite de oliva, calidad del sitio web, responsabilidad social corporativa, fSQCA.

CLAVES ECONLIT: L25, M15, 013, 033, Q01.

Cómo citar este artículo / How to cite this article: BERNAL, E., MOZAS, A., FERNÁNDEZ, D., MEDINA, M.J.

\& PUENTES, R. (2019): "Calidad de los sitios web en el sector agroalimentario ecológico y sus factores explicativos: el papel del cooperativismo", CIRIEC-España, Revista de Economía Pública, Social y Cooperativa, 95, 95-118, DOI: 10.7203/CIRIEC-E.95.13207.

Correspondencia: Enrique Bernal Jurado, ebernal@ujaen.es; Adoración Mozas Moral, amozas@ujaen.es;

Domingo Fernández Uclés, dfucles@ujaen.es, y Raquel Puentes Poyatos, rpuentes@ujaen.es, Universidad de Jaén. Miguel Jesús Medina Viruel, mjmedina@uco.es, Universidad de Córdoba. 


\section{EXPANDED ABSTRACT}

\section{Quality of web sites in the organic agro-food sector and its explanatory factors: the role of cooperativism}

Websites whose content and design is positively evaluated by users, regardless of whether they are end consumers or companies, provide firms with a source of competitive advantage. The role of the website as an information and sales channel is especially important in sectors such as the organic produce sector, where products are marketed in a strong connotative context (Evans \& Wurster, 1999) and where consumers demand a large amount of information when making purchase decisions. Information on corporate social responsibility (CSR) is particularly well received by consumers of organic produce, in the sense that these users are more concerned with issues related to the quality and authenticity of such products than with price-related issues (Morley et al., 2000; Brunso et al., 1996).

Numerous scholars have examined the metrics that influence the effectiveness of websites as a sales channel. The technology acceptance model (TAM), proposed by Davis (1989), is the most widely adopted. According to this model, the user's perception of the utility and ease of use of the website is what conditions the effectiveness of the website. Despite a lack of consensus on the definition of the key elements of perceived utility and ease of use (Heinze \& Hu, 2006), several scholars, such as DeLone and Mclean (1992), Seddon (1997) and Yang et al. (2005), have cited quality of the system and quality of the information provided as the two main determinants. This is the perspective adopted in this study.

There is also empirical evidence of the positive relationship between website quality and firm performance (Meroño \& Soto, 2007; Moral, Mozas, Bernal, \& Medina, 2015). Moreover, Moral et al. (2015) showed that organisational characteristics such as the size, legal form and age of the firm play a role as determinants of the degree of website implementation.

Based on these arguments, the goal of this study is to analyse the extent to which Spanish organic agri-food enterprises, more specifically, organic olive oil producers, exploit the potential of the Internet as a sales channel. This goal is addressed by analysing the quality of these firms' websites, with a particular focus on the CSR information provided on these websites. The influence of certain organisational and financial variables on website quality is also studied. Accordingly, five hypotheses are stated and tested: 
H1. The size of organic agri-food enterprises directly influences the overall quality of their websites.

H2. The cooperative legal form has a negative influence on the overall quality of the websites of organic agri-food enterprises.

H3. The age of organic agri-food enterprises has a significant influence on the overall quality of their websites.

H4. Website quality positively influences the performance of organic agri-food producers.

H5. The quality of CSR information has a greater influence than does general information on the performance of organic agri-food producers.

The study covered all Spanish organic extra virgin olive oil producers with active websites (116 of 259 firms in the sector). Companies that did not provide information on the target organisational and financial variables were removed from the study. Thus, the websites of 99 companies were analysed.

Based on the factors cited in the literature as essential for a high-quality website, an index of overall website quality comprising 52 indicators was developed. This index provided a quantitative measure of website quality. This index was formed of two partial indices: an index of information quality and an index of system quality. The index of information quality comprised 35 indicators (67.31\% of all indicators) divided among indicators of general information and indicators of CSR information. The index of system quality comprised 17 indicators (32.6\% of all indicators).

Data were collected in July 2016. Each indicator was assigned a score of either 1 (presence) or 0 (absence) based on the availability of each analysed aspect. A weighted score out of 10 was calculated to compute the indices, providing a simple measure that ranged from 0 to 10 . Because the two partial indices did not consist of the same number of indicators, they were weighted by their relative contribution to the index of overall website quality to compute this overall index.

The data were analysed using several methods: descriptive statistical analysis, fuzzy-set qualitative comparative analysis (fsQCA), and Pearson correlation analysis. The fsQCA technique was used to overcome the limitation of other methods as regards the use of a small sample. The results show whether the organisational variables under study (age of the company in years, number of employees working at the company offices, company size in terms of turnover and the legal form of the company) are antecedents or causal conditions of website quality.

The results of the descriptive analysis show that the average score for the index of overall website quality was 3.73 out of 10 , with a maximum value of 7.05 and a minimum value of 1.15 . The values of the partial indices show that the websites of organic producers had greater system quality (index of system quality $=3.89$ ) than information quality (index of information quality $=3.65$ ), although both indices had scores of less than 4 out of 10 . The websites lacked quality in terms of 
usability and privacy, although they were kept updated, with a score for the index of utility of 9.19 out of 10. In partial terms, organic producers used the Internet as a means of providing general information on the firm and its products (index of general information $=5.90$ ), although they did not use the Internet to deliver this information in an attractive way (interactivity $=4.46$ ). In contrast, the use of the website as a means of transparency and CSR reporting was low (CSR index $=2.10$ ). Environmental issues represented the area for which most information was provided (2.87) because of the relationship between the environment and this type of sustainable firm.

Thus, it appears that organic producers generally use the Internet with a low level of quality. Furthermore, this level of quality is greater in terms of system quality than in terms of information quality.

The results of the fsQCA reveal the existence of two sufficient causal configurations: 1) firm size combined with absence of the cooperative legal form and 2) a young business age combined with a high number of employees. These configurations partially explain the quality of organic olive oil producers' websites. The results indicate that the parsimonious solution for this model had a coverage score of 0.5821 . This level of coverage means that this set of sufficient causal configurations explains nearly $60 \%$ of the results of the model, with a consistency score of 0.7924 .

Analysis of the main causal configurations shows an inverse relationship between the age of a company and its status as a cooperative and the quality of its website. Similarly, the analysis reveals a positive relationship between the quality of the website and the size of the company, in this case measured by the European Commission's (2006) classification of organisation size as well as the number of employees.

The coverage of these two causal configurations was 0.3746 and 0.3564 . Therefore, these causal models explain approximately $40 \%$ of the quality of the analysed companies' websites. These findings lead to the weak acceptance of the first three hypotheses, without ruling out the possible existence of other organisational and structural variables that act as determinants of website quality.

Finally, the analysis of the Pearson correlation coefficient shows the existence of a positive relationship between the indices of website quality and the companies' operating income as a measure of performance. The analysis shows a significant correlation between operating income and the overall index of quality as well as the two partial indices of quality (information quality index and system quality index). The correlation was strongest in the case of the information quality index. Therefore, the fourth hypothesis may be accepted. Notably, of the quality of the information provided on the website, the CSR information had the strongest relationship with business performance (0.301; sig. $=0.009$ ). There was no significant relationship in the case of general information. This finding leads to the acceptance of the fifth hypothesis. 


\section{CALIDAD DE LOS SITIOS WEB EN EL SECTOR AGROALIMENTARIO ECOLÓGICO Y SUS FACTORES EXPLICATIVOS: EL PAPEL DEL COOPERATIVISMO}

In conclusion, Spanish organic olive oil producers face serious issues in terms of the quality of their websites. This study did not show the existence of strong relationships between website quality and organisational variables (age, size and legal form). However, the results do indicate the existence of a significant relationship between the operating income and the partial indices of quality, more specifically, the CSR information reported on the website.

KEYWORDS: Ecological agriculture, olive oil, quality of web site, Corporate Social Responsibility, fsQCA.

\section{Introducción}

La creciente sensibilización de los consumidores hacia el deterioro medioambiental (European Commision, 1999) y hacia una alimentación sana se ha visto reflejada en sus decisiones de compra y hábitos de consumo durante las dos últimas décadas y, por el lado de la oferta, se ha reflejado en un crecimiento exponencial de la producción ecológica que ha situado al sector en uno de los más dinámicos en el ámbito agroalimentario en la actualidad.

Centrándonos en el ámbito europeo, una de las características que está definiendo el crecimiento de este mercado es su desigual distribución regional, de forma que la demanda se concentra principalmente en los países de centro y norte de Europa, mientras los mediterráneos se han especializado en la producción y posterior exportación. De esta forma, poniendo como ejemplo el caso de España, la extensión de superficie certificada dedicada a la agricultura ecológica ocupa el primer lugar en el ranking europeo y el quinto a nivel mundial (Willer y Lernoud, 2018) y muestra, además, un importante ritmo de crecimiento de la superficie dedicada a la agricultura ecológica, pasando de 11 a 43,7 millones de hectáreas en el periodo 1999-2014 (Willer y Lernoud, 2018). Sin embargo, esta situación contrasta con la que existe por el lado de la demanda: en España destaca el reducido consumo de productos ecológicos, aproximadamente 21 euros per cápita/año. Esto representa apenas un 1\% en la cesta de la compra del consumidor español (MAGRAMA, 2015). El resultado de esta situación es que más del 70 por ciento de la producción ecológica española se dirige a los mercados exteriores, principalmente a Alemania, Holanda, Francia y Reino Unido (MAGRAMA, 2012).

Son numerosos los autores que han intentado encontrar una explicación a la situación anterior. En líneas generales, existe consenso en aceptar que los principales factores inhibidores de la demanda de alimentos ecológicos en España -al igual que en otros países- son el diferencial de precios existentes entre los alimentos ecológicos y sus homónimos convencionales, la deficiente distribución escasez de puntos de venta y de variedad de la oferta- y, por último, el desconocimiento y confusión que muestra el consumidor en torno a este tipo de alimentos y que, en ocasiones, se convierte en 
fuente de desconfianza (Roitner-Schobesberger, Darnhofer, Somsook y Vogl, 2008, Hamzaoui y Zahaf, 2008). Han sido diversas las respuestas dadas por el sector frente a estos factores inhibidores, entre las que cabe destacar la intensificación de la oferta de estos productos a través de canales de venta no convencionales, como es el caso de la venta directa a través de Internet (González y Cobo, 2000). Según investigaciones recientes realizadas en el sector del aceite de oliva ecológico (Medina, Mozas, Bernal y Moral, 2014; Medina, Bernal, Mozas, Moral y Fernández, 2015), la utilización del comercio electrónico puede ser uno de los factores desencadenantes de una mayor eficiencia económica y participación activa en el comercio exterior para este sector. Por su parte, otras investigaciones han puesto su atención en la importancia de la calidad del sitio web, comprobando su relevancia en otros sector de actividad (Meroño y Soto, 2007).

Con base a lo argumentado, nuestro objetivo genérico es analizar en qué medida las empresas agroalimentarias ecológicas, en concreto las empresas del sector del aceite de oliva ecológico, están aprovechando el potencial de Internet como canal de ventas, qué factores afectan a la calidad sus sitios web y cómo influye su utilización sobre la competitividad de las mismas. Para alcanzar dichos objetivos, este artículo ha quedado estructurado como sigue: tras esta introducción, en la que se ha establecido la justificación del tema de estudio y el objetivo de investigación, se hace un recorrido por el marco teórico sobre la calidad de un sitio web y se presentan las hipótesis de trabajo. En el epígrafe tercero, se explica el método de investigación utilizado y, en el cuarto, los resultados. El trabajo termina con unas conclusiones y la bibliografía.

\section{Marco teórico e hipótesis de investigación}

\subsection{La calidad del sitio web}

Los sitios web que gocen de un diseño y contenido valorado positivamente por sus usuarios, pueden constituir para las compañías una fuente de ventaja competitiva en el desarrollo de su actividad comercial. No hay que olvidar que el sitio web es el medio a través del cual muchos potenciales clientes, empleados e inversores van a tener su primer contacto con la empresa y, por tanto, va a constituir su primera referencia a la hora de crearse una imagen de la misma (Chen y Macredie, 2005). Por lo que respecta a los usuarios actuales (clientes, proveedores, accionistas, trabajadores, etc.), la Web se convierte en un lugar donde pueden familiarizarse más con la empresa, permitiéndoles completar la información sobre aspectos tales como origen de la misma, localización, métodos de producción, compromisos asumidos con el medio ambiente, etc., adquirida a través de los canales de venta tradicionales (Yang, Cai, Zhou y Zhou, 2005). Teniendo en cuenta lo anterior, son abundantes los estudios que han intentado identificar cuáles son las métricas más adecuadas para valorar la efectividad de un sitio web como sistema de información empresarial (Heinze y Hu, 2006), habiéndose rea- 


\section{CALIDAD DE LOS SITIOS WEB EN EL SECTOR AGROALIMENTARIO ECOLÓGICO Y SUS FACTORES EXPLICATIVOS: EL PAPEL DEL COOPERATIVISMO}

lizado la mayoría de ellos bajo el marco teórico propuesto por Davis (1989): el Modelo de Aceptación de Tecnologías (Technology Acceptance Model- TAM) (Lee, Kozar y Larsen, 2003).

Este sistema teórico postula que la aceptación del uso de un determinado "Sistema de Información" depende de la existencia de una actitud previa positiva hacia su uso, actitud que a su vez está estrechamente relacionada con la creencia del usuario sobre su utilidad y facilidad de uso ${ }^{1}$. La relación causal entre dichas variables goza de suficiente apoyo empírico en la literatura (Davis, 1989; Adams, Nelson y Todd, 1992; Devaraj y Kohli, 2002). Aunque no existe unanimidad a la hora de determinar cuáles son las variables externas fundamentales a la hora de influir positivamente sobre la utilidad y facilidad de uso percibidos por un usuario (Heinze y Hu, 2006), sí existe cierto consenso a la hora de aceptar que la cantidad y variedad de información disponible en los entornos virtuales están positivamente relacionados con los niveles de satisfacción de sus usuarios (Szymanski y Hise, 2000). De esta forma, Heinze y $\mathrm{Hu}$ (2006) sugieren que la presencia de un elevado nivel de información ${ }^{2}$, de interactividad $^{3}$ y de servicios ${ }^{4}$ en la web son los elementos más determinantes de la utilidad y de la facilidad de uso percibida por sus usuarios. En este sentido, diversos autores (Cristobal, Flavián y Guinalíu, 2007; Sabiote, Frías y Castañeda, 2012) sugieren que la calidad percibida es un constructo multidimensional: diseño web, el servicio al cliente, seguridad y gestión de pedidos, y esta percepción de calidad influye en la satisfacción y fidelidad de los clientes.

Desde otra perspectiva, utilizada en nuestro estudio, autores como DeLone y Mclean (1992), Seddon (1997) y Yang et al. (2005), proponen que son la calidad, tanto del sistema como de la información suministrada, los dos principales determinantes de la percepción de utilidad y facilidad de uso de un sistema de información por parte del usuario.

La calidad del sistema haría referencia a la capacidad de la red, percibida por el usuario, para recuperar y entregar la información deseada de una manera atractiva (Yang et al., 2005), utilizándose como variables de referencia más frecuentes, la usabilidad de la web, su grado de presencia, privacidad/seguridad y su interactividad.

Por su parte, la calidad de la información vendría establecida por la utilidad de sus contenidos y por el alcance de dicha información, esto es, por su suficiencia a la hora de facilitar la toma de decisiones por parte del usuario, incluyendo variables tales como la relevancia, la extensión, la actualidad y exactitud de la información suministrada, tanto del producto, como de la propia empresa (Delone y

1.- Por facilidad de uso se entiende el grado en el que un futuro usuario espera no tener que realizar nuevos esfuerzos para realizar una compra; por su parte, por utilidad percibida entendemos la probabilidad subjetiva que tiene el futuro usuario de que, con el uso de dicho sistema, mejorará el desempeño de sus funciones en una organización.

2.- La facilidad para comprender la información suministrada, de encontrarla y la calidad de la misma influyen de manera determinante en la utilidad de la web percibida por el usuario.

3.- La posibilidad de poder interactuar con un sitio web para realizar la compra de productos, de intercambiar con la compañía o con otros usuarios información sobre los mismos o de poder elegir la forma de pago elevan la satisfacción del usuario.

4.- Aspectos tales como la prestación de servicios de atención al usuario, la presencia de $F A Q$ o el suministro online de manuales de uso también elevan la utilidad percibida según los estudios realizados con consumidores online. 
Mclean, 1992; Doll, Weidong y Torkzadeh, 1994). En el caso concreto de la producción ecológica o sostenible, otro tipo de información que está influyendo en las decisiones de compra de los consumidores es la relativa a su responsabilidad social. La investigación en el área de marketing muestra que la Responsabilidad Social Corporativa (en adelante, RSC) tiene influencia positiva sobre los consumidores (Bigné, Chumpitaz, Andreu y Swaen, 2005) y que éstos prefieren los productos de empresas que invierten en acciones de protección del medio ambiente y mantienen un comportamiento responsable con la sociedad (Zaman, Yamin y Wong, 1996).

\subsection{Calidad del sitio web y su importancia en la comercialización de productos agroalimentarios ecológicos}

La calidad del sitio web puede ser especialmente relevante para alcanzar el éxito en la comercialización online de productos que poseen un fuerte contexto connotativo, como es el caso de los agroalimentarios ecológicos (Evans y Wurster, 1999). Esto es así por dos motivos principales: primero, al mayor requerimiento de información por parte de los consumidores de estos productos $y$, en segundo lugar, por la escasa información que se ha suministrado sobre los mismos a través de los canales de venta tradicionales. Efectivamente, diversos autores apuntan a que el perfil del consumidor ecológico puede incluirse en el grupo de consumidores racionales, conservadores y aventureros, caracterizados por preocuparse de atributos del producto alimenticio relacionados con la calidad y la autenticidad del mismo, más que por su precio (Morley, Sparkes, Thomas, 2000; Brunso, Grumert y Bredahl, 1996). Por ello, este perfil de consumidor se preocupa de buscar alternativas a los productos que son ofrecidos en masa en los supermercados, dirigiéndose a otros canales de venta alternativos (Pickernall et al., 2004). En esta línea, los canales de ventas tradicionales no han sabido dar respuestas a esta mayor demanda de información, obligando a los consumidores de productos ecológicos a enfrentarse a una situación de desinformación y confusión en su elección de compra (RoitnerSchobesberger et al., 2008, Hamzaoui y Zahaf, 2008).

Autores como Alonso (2005) y Vega (2011) señalan que el desconocimiento de los productos ecológicos y de sus atributos es uno de los mayores hándicaps en la expansión de su demanda, proponiendo como respuesta hacer campañas de comunicación. De hecho, Vega (2011) indica que son dos los grandes factores que han estimulado la demanda de productos ecológicos: la creciente preocupación por la salud y la dieta alimenticia, y el deterioro medioambiental que se está produciendo en los últimos años (Gil, Gracias y Sánchez, 2000), aspectos éstos que generan una elevada demanda de información. En este sentido, autores como Rivera y Brugarolas (2003) también reivindican esfuerzos en comunicación para incrementar la demanda de estos productos.

Ante esta situación, estudios como los de Baourakis (2004) indican que Internet provee del mejor canal de información para productos ecológicos, debido a su gran potencial informativo y comercial. Ello justifica la necesidad de que el sector de empresas de productos ecológicos preste una especial atención al desarrollo e incorporación de Internet en su actividad comercial (Simmons, Durkin, 


\section{CALIDAD DE LOS SITIOS WEB EN EL SECTOR AGROALIMENTARIO ECOLÓGICO Y SUS FACTORES EXPLICATIVOS: EL PAPEL DEL COOPERATIVISMO}

McGowan y Armstrong, 2007). No obstante, pese a las ventajas señaladas, el éxito de Internet como canal de venta pasa por la capacidad que posea el sitio web institucional para influir positivamente en la impresión que el usuario se cree de la compañía, pues de ésta puede depender su decisión final de mantener con la misma una relación a largo plazo (Van Der Heijden, Verhagen y Creemers, 2003). Dicha impresión viene condicionada por el potencial de la información suministrada a través de la web para compensar la ausencia en muchos casos de contacto personal entre agentes, así como generar la confianza suficiente entre los mismos (McKinney, Yoon y Zahedi, 2002). Para Salo y Karjaluototrust (2007), la confianza es un factor clave para el éxito de las transacciones online.

\subsection{Hipótesis de investigación}

Existen estudios que relacionan las características organizativas con la implementación de las Tecnologías de la Información y la Comunicación (en adelante, TICs) en la empresa. Cáceres, Guzmán y Rekowski (2011) estudian aspectos diferenciales en la implantación dependiendo del sector de actividad. La importancia del tamaño de la empresa en la adopción de las TICs ha tenido un largo debate en muchos estudios (Mehrtens, Cragg y Mills, 2001; Meroño y Soto, 2007, entre otros). Hay estudios que confirman la relación positiva entre el tamaño de la empresa y la implementación de un sitio web (Teo y Pian, 2004; Ellis-Chadwick, Doherty y Hart, 2002). Concretamente Zhu, Kraemer y Xu (2003) indican que las grandes empresas, (medidas a través del número de trabajadores) son más propensas a la adopción de las TICs. Teo y Pian (2004) también demuestran una relación positiva entre ser gran empresa y desarrollar más el sitio web. En base a estas argumentaciones teóricas, plantemos la siguiente hipótesis:

H1. El tamaño organizacional de las empresas agroalimentarias de producción ecológica influye, de manera directa, en el nivel calidad global de la web de las empresas.

Respecto a la relación de la forma jurídica de la empresa y su eficiencia, en la actualidad, no abundan estudios. No obstante, existe una serie de indicadores que apuntan a una posición rezagada, desde el punto de vista comercial, de las sociedades cooperativas con respecto a las sociedades de tipo capitalista. Al respecto, se ha contrastado una menor presencia activa de las empresas cooperativas en el comercio exterior (Medina et al., 2014); falta de orientación al mercado (Fayós y Calderón, 2013); 0 su menor utilización de las TICs (Fernández, Bernal, Mozas, Medina y Moral, 2015). La rigidez financiera de las empresas no comerciales, principalmente cooperativas (Coque, 2008), afecta a su capacidad para invertir en un sitio web de calidad. Así, y tomando como base las investigaciones anteriores, plantemos la siguiente hipótesis de investigación.

H2. La forma jurídica cooperativa tiene una influencia negativa en el nivel calidad global de la web de las empresas agroalimentarias de producción ecológica. 
Tampoco son frecuentes los trabajos que estudian, de manera directa, la antigüedad y la eficiencia de la empresa. En esta línea, Medina et al. (2015) identifican una relación negativa entre ambas variables. Por su parte, existen algunos trabajos de investigación que, de manera indirecta, apuesta por una relación positiva entre éstas, a través del efecto experiencia en los mercados internacionales (Zahra, Ireland y Hill, 2000; Autio. Sapenza y Almeida, 2000) o mediante el uso de las redes sociales virtuales (Mozas, Bernal, Medina y Fernández, 2016a).

Por su parte, esta situación de indefinición con respecto al efecto de la antigüedad de la empresa también existe en estudios que se han centrado en su carácter innovador. Así, algunos autores señalan que la experiencia provoca una actitud menos receptiva sobre la innovación (Pavitt, 2003). Por el contrario, otros autores como Chesbrough (2005) señala que la antigüedad de la empresa es un factor determinante para que ésta se adapte a las innovaciones de su entorno, en parte debido a la creación de una red de stakelholders a lo largo de los años de actividad de la empresa. Con base en estas argumentaciones teóricas plantemos nuestra tercera hipótesis de investigación.

H3. La antigüedad de las empresas agroalimentarias de producción ecológica tiene una influencia significativa en el nivel calidad global en su sitio web.

Son diversas las razones que sugieren la existencia de una relación positiva entre la calidad de una web corporativa y los rendimientos de las empresas de producción ecológica que la posean. Desde la perspectiva de la demanda, es importante destacar que las características que definen el perfil del usuario de Internet (Ministerio de Industria, Turismo y Comercio, 2011) son básicamente idénticas a las que corresponden al consumidor de productos ecológicos: jóvenes (Fraj y Martínez, 2002); con un nivel de estudios elevado (Minetti, 2002, Fraj y Martínez, 2002); y con elevados niveles de renta (Minetti, 2002; Balderjahn, 1988). Este segmento de consumidores, tan atractivo comercialmente, puede verse atraído por las empresas ecológicas a través de su web corporativa, debido a que su potencial informativo y comunicativo es capaz de dar respuesta a la mayor demanda de información de los consumidores de productos ecológicos.

Por su parte, desde el lado de la oferta, Bickerton, Bickerton y Pardesi (2000) pusieron de manifiesto el potencial de Internet para reducir los costes de transacción debido, fundamentalmente, a que hace más fácil la elección de los consumidores entre diferentes productos, incrementando la competencia global entre entidades, y reduciendo los gastos generales de producción. Respecto a esta última cuestión, es abundante la literatura que señala que las TICs tienen el potencial de reducir los costes de transacción entre actividades y de reducir el riesgo inherente a cada transacción (Strader y Shaw, 1997; Steinfeld, Kraut y Plummer, 1997, entre otros), así como de mejorar la eficiencia en las actividades de la cadena de valor (Evans y Wurster, 1997; Ghosh, 1998).

Por otro lado, frente al problema de la diseminación de la oferta a la que se enfrenta el sector de producción ecológica, Internet proporciona economías de alcance (Dholakia y Kshetri, 2004) al mejorar la cooperación interempresarial y al abrir vías que facilitan nuevos negocios e incluso incrementan 


\section{CALIDAD DE LOS SITIOS WEB EN EL SECTOR AGROALIMENTARIO ECOLÓGICO Y SUS FACTORES EXPLICATIVOS: EL PAPEL DEL COOPERATIVISMO}

la penetración de Internet en las sociedades locales, al elevar la densidad de las redes de cooperación en las que se incluyen las empresas (OCDE, 1998). En este sentido, Meroño y Soto (2007) y Moral, Mozas, Bernal y Medina (2015) analizaron la influencia de la calidad del sitio web de la empresa en los resultados obtenidos por ésta, encontrando evidencias de una relación positiva entre ambas variables. Con base en estas argumentaciones formulamos nuestra cuarta hipótesis:

H4. La calidad del sitio web influye de forma positiva sobre el rendimiento de las empresas de producción ecológica.

Además, existen evidencias empíricas de una relación positiva entre la RSC y los resultados financieros obtenidos por las empresas (Balbanis, Phillips y Lyall, 1998; Orlitzky, 2001). Ello indica que la asunción por parte de las compañías de sus responsabilidades a nivel social y medioambiental es premiada por sus grupos de interés, lo que facilita la consolidación de la posición competitiva de la empresa en el mercado y la generación de mayores beneficios (Smith, 2003; Garrigues y Trulleque, 2008). Estos estudios nos llevan a plantear la siguiente hipótesis de investigación.

H5. La calidad de la información sobre RSC tiene mayor influencia sobre los rendimientos de las empresas ecológicas que la calidad de la información general.

\section{Metodología}

El presente estudio se realizó sobre el total de empresas españolas productoras de aceite de oliva virgen extra ecológico, algunas con actividad diversificada. Después de definir el censo total (259 empresas), el primer paso fue localizar el sitio web de todas las empresas de producción ecológica a través de Internet. En total se identificaron 116 organizaciones con sitio web propio, no considerándose aquellas que se hospedaban o se anunciaban a través de terceros. El resto, o bien estaban en construcción, o bien no poseían sitio web. También fueron descartadas aquellas empresas que no presentaban información disponible sobre las variables organizativas y los datos financieros objeto de estudio. En consecuencia, fueron analizadas un total de 99 organizaciones.

Para la obtención de los datos se aplicó un checkpoint con un total de 52 ítems, clasificados en dos dimensiones, calidad de la información (Cl) y calidad del sistema (CS). Respecto a la primera dimensión, ésta se divide a su vez en calidad de la información general y calidad de la información sobre RSC. La dimensión Cl aglutina 35 indicadores (67,31 por ciento del total) y la dimensión CS 17 indicadores (32,6 por ciento), tal y como se muestra en la tabla 1. De acuerdo con los estudios de McKinney et al. (2002), Yang et al. (2005) y Witell y Löfgren (2007) estas variables permitirán obtener una valoración objetiva de la calidad de la website de las empresas analizadas. 


\section{Tabla 1. Ítems utilizados en el análisis}

\begin{tabular}{|c|c|c|}
\hline \multicolumn{2}{|c|}{ ÍTEMS DE CALIDAD DE LA INFORMACIÓN (Cl) } & \multirow{2}{*}{$\begin{array}{c}\text { ÍTEMS DE CALIDAD DEL } \\
\text { SISTEMA (CS) }\end{array}$} \\
\hline Información General & Información de RSC & \\
\hline $\begin{array}{l}\text { Utilidad: } \\
\text { Sitio web actualizado } \\
\text { Sitio web totalmente construido } \\
\text { Información sobre la empresa: } \\
\text { Origen/historia } \\
\text { Localización } \\
\text { Información del presidente/ } \\
\text { junta directiva } \\
\text { Información sobre el entorno } \\
\text { Pertenencia a denominación } \\
\text { de origen } \\
\text { Información sobre el producto: } \\
\text { Técnicas de producción } \\
\text { Control de calidad } \\
\text { Cuidado del medio ambiente } \\
\text { Catálogo de productos } \\
\text { Precios de los productos } \\
\text { Descuentos por compras online } \\
\text { Recomendaciones sobre el } \\
\text { consumo del producto } \\
\text { Información sobre el tiempo de } \\
\text { entrega }\end{array}$ & $\begin{array}{l}\text { Dimensión medioambiental: } \\
\text { Utilización de material reciclado } \\
\text { Generación de energía renovable } \\
\text { Emisión de vestidos y residuos } \\
\text { Política de protección medioambiental } \\
\text { Acciones de I+D para protección del } \\
\text { medioambiente } \\
\text { Producción ecológica } \\
\text { Premios y Reconocimientos de producción } \\
\text { ecológica } \\
\text { Dimensión social } \\
\text { Información sobre } n^{\circ} \text { y tipo de trabajadores } \\
\text { en la empresa } \\
\text { Cursos de formación para empleados } \\
\text { Política de Recursos humanos (conciliación, } \\
\text { beneficios sociales, ...) } \\
\text { Política de seguridad alimentaria } \\
\text { Código de conducta hacia la cadena de } \\
\text { producción } \\
\text { Patrocinio de actividades } \\
\text { Premios por acciones responsables } \\
\text { Dimensión económica } \\
\text { Datos económicos } \\
\text { Inversiones en I+D+i } \\
\text { Acceso a las cuentas anuales } \\
\text { Aplicaciones de resultados a fondos de } \\
\text { educación y promoción } \\
\text { Estructura organizativa } \\
\text { Gestión socialmente responsable }\end{array}$ & $\begin{array}{l}\text { Usabilidad: } \\
\text { Links } \\
\text { Banners } \\
\text { Sonido } \\
\text { Vídeo } \\
\text { Mapas web } \\
\text { Privacidad: } \\
\text { Información sobre la seguridad } \\
\text { en los pagos } \\
\text { Información sobre la privacidad } \\
\text { de la información } \\
\text { Interactividad: } \\
\text { Registro de clientes } \\
\text { Cookies } \\
\text { Correo electrónico } \\
\text { Teléfono } \\
\text { Preguntas frecuentes } \\
\text { Seguimiento de los pedidos } \\
\text { Acceso restringido a los socios } \\
\text { Información ofrecida en varios } \\
\text { idiomas } \\
\text { Tienda electrónica } \\
\text { Acceso a redes sociales de la } \\
\text { empresa }\end{array}$ \\
\hline
\end{tabular}

FUENTE: Elaboración propia.

La recopilación de esta información se llevó a cabo durante julio de 2016. Cada atributo o cualidad valorada fue valorada con el valor 1 (presencia) 00 (ausencia), considerando la disponibilidad del aspecto analizado. 
Hemos elaborado un Índice de Calidad Global de la website (en adelante ICG) con los 52 indicadores estudiados, que nos permite obtener una medida cuantitativa de la calidad de la website. Una vez medido el nivel de seguimiento de cada uno de los indicadores, se han calculado dos índices de calidad parciales vinculados a cada una de las dimensiones analizadas: un Índice de Calidad de la Información (ICI) y un Índice de Calidad del Sistema (ICS). Estos índices se han calculado siguiendo a Mozas, Puentes y Bernal (2010) y Chaves, Mozas, Puentes y Bernal (2011), aplicando la siguiente fórmula (fórmula 1):

$$
\begin{gathered}
\text { Fórmula } 1 \\
\mid \mathrm{Cl}=\frac{\sum \text { Puntos obtenidos en cada indicador }}{\sum \text { Puntos totales que podría obtener en cada indicador }} \times 10
\end{gathered}
$$

Este indicador parcial se ha ponderado sobre 10 para obtener una medida simple del mismo comprendida entre 0 y 10 . Para el cálculo del índice global o ICG se procede sumando los índices parciales una vez ponderados por su peso relativo dentro del índice total, dado que no recogen el mismo número de indicadores. Así, el ICG se calcularía como sigue (fórmula 2):

\section{Fórmula 2}

$$
I C G=I C I \times 67,31 \%+I C S \times 32,69 \%
$$

Los datos se han tratado utilizando diversos métodos, como: estadística descriptiva y la técnica Fuzzy Set Qualitative Comparative Analysis (fsQCA), motivado esto por la limitación que supone, en otros métodos, el tamaño de la muestra. Además, para comprobar la realización existente entre los índices de calidad de los sitios web y los resultados económicos de las organizaciones, el estudio se ha completado con el cálculo de correlaciones de Pearson.

La técnica fsQCA es utilizada para contrarrestar la limitación de tamaño de la población estudiada, que cómo se ha indicado anteriormente ha sido de 99 empresas. La técnica Qualitative Comparative Analysis (QCA) fue creada por Ragin (1987), dando lugar a lo que hoy se conoce como Crisp Set QCA (csQCA) o QCA convencional. Desde este momento fue aumentando su utilización y fama, debido principalmente a la suficiencia de muestra de tamaño medio o bajo, una de sus ventajas más destacadas (Ragin, Shulman, Weinberg y Grand, 2003; Ragin y Rihoux, 2004).

No obstante, la técnica csQCA no estaba exenta de inconvenientes. La principal limitación de esta primera variante de la técnica QCA, para su uso en el ámbito científico, es la exclusiva utilización de variables dicotómicas. Esta limitación se solucionó con la utilización de la teoría de conjuntos difusos y Boolean logic, apareciendo así la variante fsQCA (Ragin, 2008), variante utilizada en nuestra investigación. Esta nueva variante permite la utilización de variables continuas y por intervalos. Para la inclu- 
sión de estos nuevos tipos de variables es necesario realizar una calibración en las mismas. El objetivo de la calibración es convertir las variables en variables fuzzy, asignando valores comprendidos entre 0 y 1. Esta asignación se realizará en función del grado de afiliación dentro de la categoría (Schneider, Schulze-Bentrop y Paunescu, 2010; Woodside, 2013).

El método ofrece para un resultado concreto $(Y)$, una 0 varias combinaciones causales suficientes, por ejemplo: $\mathrm{X} 1^{*} \sim \mathrm{X} 2^{*} \mathrm{X} 3$, donde: $\mathrm{X} 1, \mathrm{X} 2$ y X3 son antecedentes 0 causas; * la unión de las causas y $\sim$ la ausencia, negación o relación inversa entre el antecedente y el resultado (Mozas, Moral, Medina y Bernal, 2016b).

Como variable dependiente o resultado se ha utilizado el índice de calidad global de los sitios web (ICG) de las empresas de nuestra muestra, obteniéndose según la fórmula 2. Esta variable se ha calibrado con el programa fsQCA 2.0. Por su parte, en la tabla 2 se muestran las variables que se han utilizado como antecedentes o condiciones causales.

\section{Tabla 2. Variables utilizadas en el modelo fsQCA y su calibración}

\begin{tabular}{|l|c|c|}
\hline \multicolumn{2}{|l|}{ VARIABLE } & CALIBRACIÓN \\
\hline Antigüedad de la empresa en años & Antig & Calibrada con fsQCA 2.0 \\
Número de empleados en oficina de la empresa & Empl & Calibrada con fsQCA 2.0 \\
Tamaño empresarial. Tipología* & Tam.tipo & Calibrada con fsQCA 2.0 \\
Forma jurídica con la que opera la empresa & F_Jur & Variable dicotómica** \\
\hline
\end{tabular}

* Calibrada según la clasificación de la European Commission (2006) en: 1: microempresa; 2: empresa pequeña; 3: mediana empresa y 4: empresa grande; ${ }^{* *}$ Calibrada de la siguiente forma: 0 (forma jurídica no cooperativa), 1 (forma jurídica cooperativa). FUENTE: Elaboración propia.

\section{Resultados}

\section{1. Índice de Calidad Global (ICG)}

Una vez analizados los indicadores de forma individualizada, se calculó un indicador sintético de la situación del sector. En la tabla 3, se muestran los principales estadísticos descriptivos tanto de los índices parciales como del índice global de calidad que caracterizan a las entidades estudiadas. 


\section{Tabla 3. Estadísticos descriptivos Índice de Calidad de la Información y del Sistema en web}

\begin{tabular}{|l|ccccc|}
\hline & N & Media & Desv.Típ. & Max. & Min. \\
\hline Índice de Calidad de la Información (ICI) & 99 & 3,65 & 1,38 & 7,39 & 1,01 \\
Índice de Información general & 99 & 5,90 & 1,25 & 8,40 & 1,25 \\
Utilidad & 99 & 9,19 & 1,98 & 10,00 & 0,00 \\
Información empresa & 99 & 7,47 & 1,82 & 10,00 & 1,82 \\
Información producto & 99 & 4,10 & 1,44 & 7,00 & 0,00 \\
Índice de Información de RSC & 99 & 2,10 & 2,06 & 7,50 & 0,00 \\
Dimensión medioambiental & 99 & 2,87 & 3,02 & 10,00 & 0,00 \\
Dimensión social & 99 & 1,54 & 1,98 & 8,57 & 0,61 \\
Dimensión económica & 99 & 1,53 & 2,07 & 8,33 & 0,00 \\
Índice de Calidad del Sistema (ICS) & 99 & 3,89 & 1,44 & 7,65 & 1,18 \\
Usabilidad & 99 & 2,79 & 2,17 & 8,00 & 0,00 \\
Privacidad & 99 & 3,79 & 4,58 & 10,00 & 0,00 \\
Interactividad & 99 & 4,46 & 1,56 & 9,00 & 1,56 \\
Índice de Calidad Global (ICG) & 99 & 3,73 & 1,15 & 7,05 & 1,15 \\
\hline
\end{tabular}

FUENTE: Elaboración propia.

Sobre la base de los índices parciales, el ICG alcanza 3,73 puntos de media sobre 10, con un valor máximo del índice que se sitúa en 7,05 y el valor mínimo en 1,15. Los valores de los índices parciales muestran como la website de las empresas de producción ecológica presentan una mayor calidad del sistema (ICS = 3,89 puntos) que de la información ( $\mathrm{ICl}=3,65$ puntos), siendo ambos inferiores a un valor de 4 sobre 10. Adolecen de calidad en cuanto a usabilidad y privacidad de la web, aunque mantienen la misma actualizada, siendo el índice de utilidad de 9,19 puntos sobre 10. En términos parciales, las empresas de producción ecológica utilizan Internet como medio de información general sobre la empresa y su producto (Índice Información General = 5,90 puntos), aunque no la utilizan para entregar esta información de una manera atractiva (Interactividad $=4,46$ puntos). Por otro lado, se puede apreciar que la utilización de la web como medio de transparencia o de comunicación en términos de RSC es muy baja (Índice de RSC =2,10 puntos), siendo el ámbito medioambiental del que más se informa (2,87 puntos), por la relación entre éste y el tipo de empresa sostenible.

En consecuencia, podemos decir que, en términos generales, las empresas de producción ecológica utilizan Internet con un nivel de calidad baja. Además, también destaca que este nivel de calidad es mayor en la calidad del sistema (ICS) frente a la calidad de la información (ICI). 


\subsection{Resultados del análisis multivariante}

Con el objetivo de determinar si los factores propuestos en este estudio (tamaño, forma jurídica y antigüedad) influyen en la calidad de los sitios web, y ante la limitación del tamaño de la población estudiada, se ha aplicado la técnica fsQCA. Una vez realizado el análisis con el programa fsQCA 2.0, y como se muestra en la tabla 4, se comprueba la existencia de 2 combinaciones causales suficientes [(Tam.tipo* F_Jur) y ( antig*empl)], que explican parte de la calidad de los sitios web de las empresas de aceite de oliva ecológico.

Los resultados del análisis (tabla 4) indican que la solución final de este modelo en su solución parsimoniosa tiene una tasa de cobertura de 0.5821 , por lo que el conjunto de combinaciones causales suficientes explica casi el 60 por ciento del resultado del modelo, con un índice de consistencia de 0.7924 .

\section{Tabla 4. Resultados Fuzzy Set QCA}

\begin{tabular}{|l|ccc|}
\hline Configuración causal & Tasa de cobertura total & Tasa de cobertura exclusiva & Índice de consistencia \\
\hline Tam.tipo* $\sim$ F_Jur & 0.374627 & 0.156418 & 0.808634 \\
antig*empl & 0.356418 & 0.106567 & 0.806757 \\
antig $^{*} \sim$ empl*F_Jur & 0.100896 & 0.069254 & 0.908602 \\
\hline Tasa de cobertura de la solución: 0.582090 & & \\
Índice de consistencia de la solución: 0.792361 & & \\
\hline
\end{tabular}

FUENTE: Elaboración propia.

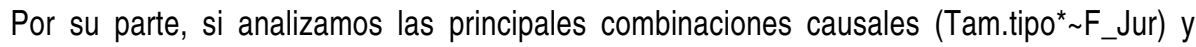
( antig*empl), vemos que existe una relación inversa entre la antigüedad de la organización y la forma jurídica cooperativa con la calidad de su sitio web. Del mismo modo, existe una relación positiva entre dicha calidad del sitio web y el tamaño de la organización, en esta ocasión medido tanto por la clasificación de la European Commission (2006) de dimensión de organizaciones (Variable: "Tam.tipo") como por el número de empleados (variable: "empl").

Las tasas de cobertura de estas combinaciones causales son de 0.3746 y 0.3564 . Por tanto, estos modelos causales explican en torno al 40 por ciento de la calidad del sitio web de las empresas estudiadas. Estos resultados nos llevan a aceptar las tres primeras hipótesis de estudio, no rechazando la posible existencia de otras variables organizativas y estructurales como también determinantes de la calidad del sitio web.

Por otro lado, para corroborar o no nuestra cuarta hipótesis, la influencia del índice de calidad total y los índices de calidad parciales sobre las variables económicas, hemos comprobado, como paso previo, la existencia de normalidad, autocorrelación y homocedasticidad de los residuos y multicoli- 


\section{CALIDAD DE LOS SITIOS WEB EN EL SECTOR AGROALIMENTARIO ECOLÓGICO Y SUS FACTORES EXPLICATIVOS: EL PAPEL DEL COOPERATIVISMO}

nealidad de las variables independientes (Hengging, Krishna, Yanging, Yanging, 2011). Para asegurar la hipótesis de normalidad ha sido necesario aplicar la transformación de Box-Cox (Hengging et al. 2011) a las variables económicas (ingresos de explotación: Y1 y resultados del ejercicio antes de impuestos: Y2), siendo solo la variable Y1 la que cumple con esas tres hipótesis, por lo que el estudio se va a centran en la influencia de los índices de calidad de la información (ICI) y calidad del sistema (ICS) (variables independientes) sobre los Ingresos de explotación (Y1).

Posteriormente se realizó un análisis de correlación de Pearson entre los distintos índices de calidad de los sitios web y los ingresos de explotación de las empresas. Los resultados se exponen en la tabla 5. En ella puede apreciase la existencia de una correlación significativa entre los ingresos de explotación y el índice de calidad global (ICG) y los dos índices de calidad parciales (ICl e ICS), siendo más fuerte en el caso del índice de calidad de la información (ICI). Por tanto, puede ser aceptada la cuarta hipótesis de investigación. También cabe resaltar que, dentro de la calidad de la información del sitio web, es la información sobre RSC la que se relaciona con los resultados empresariales $(0,301$; Sig. 0,009), no existiendo una relación significativa en el caso de la información general. Ello nos lleva a aceptar nuestra quinta hipótesis de investigación.

\section{Tabla 5. Correlaciones respecto a los ingresos de explotación}

\begin{tabular}{|l|c|c|}
\hline & Correlación de Pearson & Sig. \\
\hline Índice de Calidad Global (ICG) & $0,371^{* *}$ & 0,001 \\
Índice de Calidad de la Información (ICI) & $0,314^{* *}$ & 0,006 \\
Índice de Información general & 0,159 & 0,173 \\
Índice de Información RSC & $0,301^{* \star}$ & 0,009 \\
Índice de Calidad del Sistema (ICS) & $0,293^{*}$ & 0,011 \\
\hline
\end{tabular}

${ }^{*}$ Correlación significativa al 90 por ciento de nivel de confianza.

${ }^{\star *}$ Correlación significativa al 95 por ciento de nivel de confianza.

FUENTE: Elaboración propia. 


\section{Conclusiones}

El sitio web ofrece a las empresas un espacio en el que desarrollar gran parte de los procesos de comunicación con sus clientes y un nuevo canal de venta capaz de difuminar la importancia de la distancia física que separa a compradores y vendedores. Ello convierte a estas plataformas en canales de venta especialmente atractivos para sectores, como el agroalimentario ecológico, que tradicionalmente han afrontado importantes deficiencias comerciales relacionadas fundamentalmente con la escasez de información y de puntos de venta en el mercado. Partiendo de estas premisas, los objetivos de este trabajo son: examinar en qué medida las empresas españolas del sector de la agricultura ecológica están aprovechando el sitio web como canal de venta, identificar los factores que explican la calidad de estas plataformas virtuales y estudiar la relación de dicha calidad con los rendimientos empresariales.

Los resultados obtenidos en este estudio permiten afirmar que las empresas españolas de producción ecológica de aceite de oliva tienen un importante déficit respecto a la calidad de sus sitios web. Esta situación se agrava, según las diferentes pruebas paramétricas realizadas, en el caso de las empresas que actúan bajo la forma jurídica cooperativa y las de mayor antigüedad y mejora entre las empresas de mayor tamaño. Además, los resultados extraídos en esta investigación reflejan la existencia de una relación significativa entre los resultados de explotación y los índices parciales de calidad del sitio web. Cabe destacar, que es la información sobre RSC la que se relaciona con más intensidad con los resultados empresariales. Esto corrobora la importancia que en la actualidad tienen estas plataformas virtuales para el avance comercial de las organizaciones y de informar a través de ellas las principales prácticas de RSC.

Este trabajo es uno de los pocos estudios que, con datos obtenidos a partir de análisis directos de la website de las empresas de productos ecológicos, permite obtener una visión general sobre la calidad de las mismas. Los resultados obtenidos se han obtenido de la población total de empresas españolas ecológicas del sector oleícola, por lo que son significativos y las deficiencias que han aflorado deben de alentar a las empresas del sector, en particular a las cooperativas, a invertir en la calidad de su sitio web, instrumento imprescindible en el diseño e implementación de estrategias de expansión o diversificación de su negocio.

Las futuras líneas de investigación deben centrarse en estudiar la existencia de variables estructurales y organizacionales que, junto con los avances en la adopción de las TICs y del comercio electrónico, expliquen la mejora comercial de las empresas del sector de producción ecológica. 


\section{Referencias bibliográficas}

ADAMS, D.A., NELSON, R. \& TODD, P. (1992): "Perceived usefulness, ease of use and usage of information technology: a replication", MIS Quarterly, 16(2), 227-247, doi: 10.2307/249577.

ALONSO, A. (2005): "Caracterización del mercado de productos ecológicos frescos. El caso de Granada", Distribución y Consumo, 84, 65-75.

AUTIO, E., SAPENZA, H.J. \& ALMEIDA, J.G. (2000): "Effects of age at entry knowledge intensity and imitability on international growth", The Academy of Management Journal, 43(5), 909-924. doi: $10.2307 / 1556419$.

BALBANIS, G., PHILLIPS, H.C. \& LYALL, J. (1998): "Corporate social responsibility and economic performance in the top British companies: are they linked?", European Business Review, 98(1), 2544, doi: 10.1108/09555349810195529.

BALDERJAHN, L. (1988): "Personality variables and environmental attitudes as predictors of ecologically responsible consumption patterns", Journal of Business Research, 17(1), 51-56, doi: 10.1016/0148-2963(88)90022-7.

BAOURAKIS, G. (2004): Marketing trends for organic food in the 21st century (Vol. 3), World Scientific.

BASARAN, B., KONYALI, S. \& ORAMAN, Y. (2018): "An overview of organic producer organizations in Turkey", New knowledge Journal of science, 10(2), 45-54.

BICKERTON, P., BICKERTON, M. \& PARDESI, U. (2000): Cybermarketing, Butterworth-Heinemann, Oxford.

BIGNE, E., CHUMPITAZ, R., ANDREU, L. \& SWAEN, V. (2005): "Percepción de la responsabilidad social corporativa: un análisis cross-cultural", Universia Business Review, 5, 14-27.

BRUNSO, K., GRUMERT, F.G. \& BREDAHL, L. (1996): "An analysis of national and cross-national consumer segments using the food-related lifestyle instrument in Denmark, France, Germany and Great Britain", MAPP -Aarhus School of Business, 35, 1-53.

CÁCERES, R., GUZMÁN, J. \& REKOWSKI, M. (2011): "Firms as source of variety in innovation: influence of size and sector", International Entrepreneurship and Management Journal, 7(3), 357-372, doi: 10.1007/s11365-011-0198-8.

CRISTOBAL, E., FLAVIÁN, C. \& GUINALÍU, M. (2007): "Perceived e-service quality (PeSQ): Measurement validation and effects on consumer satisfaction and web site loyalty", Managing Service Quality, 17(3), 317-340, doi: 10.1108/09604520710744326. 
CHAVES, R., MOZAS, A., PUENTES, R. \& BERNAL, E. (2011): "E-corporate social responsibility in socially responsible firms: the case of Spanish firms", The Service Industries Journal, 31(12), 118, doi: 10.1080/02642069.2011.545880.

CHEN, S. \& MACREDIE, R. (2005): "The assessment of usability of electronic shopping a heuristic evaluation", International Journal of Information Management, 25(6), 516-532, doi: 10.1016/j.jijinfomgt.2005.08.008.

CHESBROUGH, H. (2005): Open innovation, Boston, Harvard Bussiness School Press.

COQUE, J. (2008): "Puntos fuertes y débiles de las cooperativas desde un concepto amplio de gobierno empresarial", REVESCO, Revista de Estudios Cooperativos, 2(95), 65-93.

DAVIS, F.D. (1989): "Perceived usefulness, perceived ease of use and user acceptance of information technology", MIS Quarterly, 13(3), 319-340, doi: 10.2307/249008.

DELONE, W. \& MCLEAN, E. (1992): "Information systems success: the quest for the dependent variable", Information on Systems Research, 3(1), 60-95, doi: 10.1287/isre.3.1.60.

DEVARAJ, S. \& KOHLI, M. (2002): "Antecedents of B2C channel satisfaction and reference: Validating e-Commerce metrics", Information System Research, 13(3), 316-333, doi: 10.1287/isre.13.3.316.77.

DHOLAKIA, R. \& KSHETRI, N. (2004): "Factors impacting the adoption of the Internet among SMEs", Small Business Economics, 23(4), 311-322, doi: 10.1023/B:SBEJ.0000032036.90353.1f.

DOLL, W., WEIDONG, X. \& TORKZADEH, G. (1994): "A confirmatory factor analysis of the end-user computing satisfaction instrument", MIS Quarterly, 18(4), 453-461, doi: 10.2307/249524.

ELLIS-CHADWICK, F., DOHERTY, N. \& HART, C. (2002): "Signs of change? A longitudinal study of Internet adoption in the UK retail sector", Journal of Retailing and Consumer Services, 9(2), 7180, doi: 10.1016/S0969-6989(01)00030-3.

EUROPEAN COMMISSION (1999): What do Europeans think about the environment, Eurobarometer 51.1, Luxemburg.

EUROPEAN COMMISSION (2006): La nueva definición de pyme, Publicaciones Empresa e Industria.

EVANS, P.B. \& WURSTER, T.S. (1997): "Strategy and the new economics of information", Harvard Business Review, 75(5), 71-82.

FAYOS, Y. \& CALDERON, H. (2013): "Principales problemas de internacionalización de las cooperativas agroalimentarias españolas", REVESCO, Revista de Estudios Cooperativos, 111, $32-59$.

FERNÁNDEZ, D., BERNAL, E., MOZAS, A., MEDINA, M.J. \& MORAL, E. (2015): "El sector cooperativo oleícola y el uso de las TIC: un estudio comparativo respecto a otras formas jurídicas", REVESCO, Revista de Estudios Cooperativos, 120, 53-75.

FRAJ, E. \& MARTÍNEZ, E. (2002): Comportamiento del consumidor ecológico, Esic Editorial, Madrid. 
GARRIGUES, A. \& TRULLEQUE, F. (2008): "Responsabilidad social corporativa: ¿papel mojado 0 necesidad estratégica?", Harvard - Deusto Business Review, 164, 19-36.

GHOSH, S. (1998): "Making business sense of the Internet", Harvard Business Review, 76(2), 126135.

GIL, J.M., GRACIAS, A. \& SÁNCHEZ, M. (2000): "Market segmentationand willingness top pay for organic products in Spain", International Food and Agribusiness Management Review, 3, 207-226, doi: 10.1016/S1096-7508(01)00040-4.

GONZÁLEZ, L. \& COBO, F.B. (2000): "Agricultura ecológica en España. Las estrechas claves para el éxito", Distribución y Consumo, 51, 39-55.

HAMZAOUI, L. \& ZAHAF, M. (2008): "Decision making process of community organic food consumers: an explanatory study", Journal of Consumer Marketing, 25(2), 95-104.

HEINZE, N. \& HU, Q. (2006): "The evolution of corporate web presence: a longitudinal study of large American companies", International Journal of Information Management, 26(4), 313-525, doi: 10.1016/j.ijinfomgt.2006.03.008.

HENGGING, T., KRISHNA, K.T., YANGING, H. \& YANGING, S. (2011): Developing econometrics: Statistical theories and methods with applications, Wiley-Blackwell, Chichester.

LEE, Y., KOZAR, K.A. \& LARSEN, K.R.T. (2003): "The Technology Acceptance Model: Past, present and future", Communications of the Association for Information Systems, 12(1), 752-780, doi: 10.17705/1CAIS.01250.

MAGRAMA (Ministerio de Agricultura, Alimentación y Medio Ambiente) (2012): Caracterización del mercado de productos ecológicos en los canales especialistas de venta, Madrid.

MAGRAMA (Ministerio de Agricultura, Alimentación y Medio Ambiente) (2015): Caracterización de la comercialización y distribución de productos ecológicos a través de los canales de venta especializados, Madrid.

MCKINNEY, V., YOON, K. \& ZAHEDI, F.M. (2002): "The measurement of web-customer satisfaction: an expectation and disconfirmation approach", Information Systems Research, 13(3), 296-315, doi: 10.1287/isre.13.3.296.76.

MEDINA, M.J., BERNAL, E., MOZAS, A., MORAL, E. \& FERNÁNDEZ, D. (2015): "Efficiency of organic farming companies that operate in an online environment", Custos e agronegócio online, 11(4), 264-289.

MEDINA, M.J., MOZAS, A., BERNAL, E. \& MORAL, E. (2014): "Factores determinantes para la exportación en las empresas cooperativas oleícolas andaluzas", CIRIEC-España, Revista de Economía Pública, Social y Cooperativa, 81, 241-262.

MEHRTENS, J., CRAGG, P.B. \& MILLS, A.M. (2001): "A model of Internet adoptions by SMEs", Information \& Management, 39(3), 165-176, doi: 10.1016/S0378-7206(01)00086-6. 
MERONO, A.L. \& SOTO, P. (2007): "External web content and its influence on organizational performance", European Journal of Informations Systems, 16(1), 66-80, doi: 10.1057/palgrave.ejis.3000656.

MINETTI, A.C. (2002): Marketing de alimentos ecológicos, Ediciones Pirámides y ESIC, Madrid.

MINISTERIO DE INDUSTRIA, TURISMO Y COMERCIO (2011): Análisis de datos INE 2010, Ministerio de Industria, Turismo y Comercio, Madrid.

MORLEY, A., SPARKES, A. \& THOMAS, B. (2000): "Strategic and regional initiatives in the agri-food industry in Wales", British Food Journal, 102(4), 274-89, doi: 10.1108/00070700010327689.

MORAL, E., MOZAS, A., BERNAL, E. \& MEDINA, M.J. (2015): "Efficiency and exports: Evidence from Southern European companies", Journal of Business Research, 68(7), 1506-1511, doi: 10.1016/j.jbusres.2015.01.042.

MOZAS, A., BERNAL, E., MEDINA, M.J. \& FERNÁNDEZ, D. (2016): "Factors for success in online social networks: An fsQCA approach", Journal of Business Research, 69(11), 5261-5264, doi: 10.1016/j.jpusres.2016.04.122.

MOZAS, A., MORAL, E., MEDINA, M.J. \& BERNAL, E. (2016): "Manager's educational background and ICT use as antecedents of export decisions: A crisp set QCA analysis", Journal of Business Research, 69(11), 1333-1335, doi: 10.1016/j.jbusres.2015.10.102.

MOZAS, A., PUENTES, R. \& BERNAL, E. (2010): "La E-corporate social responsibility en las sociedades cooperativas andaluzas", CIRIEC-España, Revista de Economía Pública, Social y Cooperativa, 67, 187-212.

OCDE (1998): The economic and social impacts of electronic commerce: preliminary findings and research agenda, OECD.

ORLITZKY, M. (2001): "Does firm size confound the relationship between corporate social performance and firm financial performance?", Journal of Business Ethics, 33(2), 167-180, doi: 10.1023/A:1017516826427.

PAVITT, K. (2003): "The process of innovation". In: Mowery, J.D. \& Nelson, R. (Eds.), Handbook on innovation, Oxford: Oxford University Press.

RAGIN, C.C. (1987): The comparative method: moving beyond qualitative and quantitative strategies, University of California Press, Berkeley.

RAGIN, C.C. (2008): Redesigning social inquiry: fuzzy sets and beyond, University of Chicago Press, Chicago.

RAGIN, C.C. \& RIHOUX, B. (2004): "Qualitative comparative analysis (QCA): state of the art and propects", Qualitative Methods, 2(2), 3-13. 
RAGIN, C.C., SHULMAN, D., WEINBERG, A. \& GRAND, B. (2003): "Complexity, generality and qualitative comparative analysis", Field Methods, 15(4), 323-340, doi: 10.1177/1525822X03257689.

RIVERA, L.M. \& BRUGAROLAS, M. (2003): "Estrategias comerciales para los productos ecológicos", Distribución y Consumo, 67, 15-22.

ROITNER-SCHOBESBERGER, B., DARNHOFER, I., SOMSOOK, S. \& VOGL, C.R. (2008): "Perceptions of organic foods in Bangkok", Thailand. Food Policy, 33(2), 112-121, doi: 10.1016/j.foodpol.2007.09.004.

SABIOTE, C.M., FRÍAS, D.M. \& CASTAÑEDA, J.A. (2012): "E-service quality as antecedent to e-satisfaction. The moderating effect of culture", Online Information Review, 36(2), 157-174, doi: 10.1108/14684521211229011.

SALO, J. \& KARJALUOTO, H. (2007): "A conceptual model of trust in the online environment", Online Information Review, 31(5), 604-621, doi: 10.1108/14684520710832324.

SCALVEDI, M.L. \& SABA, A. (2018): "Exploring local and organic food consumption in a holistic sustainability view", British Food Journal, 120(4), 749-762, doi: 10.1108/BFJ-03-2017-0141.

SCHNEIDER, M.R., SCHULZE-BENTROP, C. \& PAUNESCU, M. (2010): "Mapping the institutional capital of high-tech firms: A fuzzy-set analysis of capitalist variety and export performance", Journal of International Business Studies, 41, 246-266, doi: 10.1057/jibs.2009.36.

SEDDON, P. (1997): "A respecification and extension of the DeLone and McLean Model of IS success", Information Systems Research, 8(3), 240-253, doi: 10.1287/isre.8.3.240.

SIMMONS, G., DURKIN, M., MCGOWAN, P. \& ARMSTRONG, G. (2007): "Determinants of Internet adoption by SME agri-food companies", Journal of Small Business and Enterprise Development, 14(4), 620-640, doi: 10.1108/14626000710832730.

SMITH, N.C. (2003): "Corporate social responsibility: whether or how?", California Management Review, 45(4), 52-76, doi: 10.2307/41166188.

STEINFELD, C., KRAUT, R. \& PLUMMER, A. (1997): "The impact of interorganizational networks on buyer-seller relationships", Journal of Computer-Mediated Communications (online), 1-3, doi: 10.1111/j.1083-6101.1995.tb00168.x.

STRADER, T. \& SHAW, M.J. (1997): "Characteristic of electronic markets", Decision Support Systems, 21(3), 185-198, doi: 10.1016/S0167-9236(97)00028-6.

SZYMANSKI, D.M. \& HISE, R.T. (2000): "E-satisfaction: an initial examination", Journal of Retailing, 76(3), 309-322, doi: 10.1016/S0022-4359(00)00035-X.

TEO, T.S.H. \& PIAN, Y. (2004): "A model of web adoption", Information \& Management, 41(4), 457468, doi: 10.1016/S0378-7206(03)00084-3. 
VAN DER HEIJDEN, H., VERHAGEN, T. \& CREEMERS, M. (2003): "Understanding online purchase intentions: Contributions from technology and trust perspectives", European Journal of Information Systems, 12(1), 41-48, doi: 10.1057/palgrave.ejis.3000445.

VEGA, M. (2011): Un modelo explicativo del comportamiento del consumidor de aceite de oliva ecológico en España, Tesis doctoral, Universidad de Jaén, España.

WILLER, H. \& LERNOUD, J. (Eds.) (2018): The World of Organic Agriculture. Statistics and Emerging Trends 2018, Research Institute of Organic Agriculture (FiBL), Frick, and IFOAM - Organics International, Bonn.

WITELL, L. \& LÖFGREN, M. (2007): "Classification of quality attributes", Managing Service Quality, 17(1), 54-73, doi: 10.1108/09604520710720674.

WOODSIDE, A.G. (2013): "Moving beyond multiple regression analysis to algorithms: Calling for adoption of a paradigm shift from symmetric to asymmetric thinking in data analysis and crafting theory", Journal of Business Research, 66(4), 463-472, doi: 10.1016/j.jbusres.2012.12.021.

YANG, Z., CAI, S. ZHOU, Z. \& ZHOU, N. (2005): "Development and validation of an instrument to measure user perceived service quality of information presenting web portals", Information \& Management, 42(4), 575-589, doi: 10.1016/j.im.2004.03.001.

ZAHRA, S.A., IRELAND, R.D. \& HILL, M.A. (2000): "International expansion by new venture firms: international diversity, mode of market entry, technological learning, and performance", The Academy of Management Journal, 43(5), 925-950, doi: 10.2307/1556420.

ZAMAN, M., YAMIN, S. \& WONG, F. (1996): "Environmental consumerism and buying preference for green products". In: Proceedings of the Australian Marketing Educators Conference: Theory and Application, Adelaide, Marketing Science Centre, University of South Australian, 613-626.

ZHU, K., KRAEMER, K. \& XU, S. (2003): "Electronic Business adoption by European firms: a crosscountry assessment of facilitators and inhibitors", European Journal of Information Systems, 12(4), 251-268, doi: 10.1057/palgrave.ejis.3000475. 